\title{
Abriendo Oportunidades: Sesión-Mi plan de vida
}

Population Council

Follow this and additional works at: https://knowledgecommons.popcouncil.org/departments_sbsr-pgy

Part of the Civic and Community Engagement Commons, Family, Life Course, and Society Commons, and the Gender Equity in Education Commons How does access to this work benefit you? Let us know!

\section{Recommended Citation}

"Abriendo Oportunidades: Sesión-Mi plan de vida." Guatemala City: Population Council, 2016. 


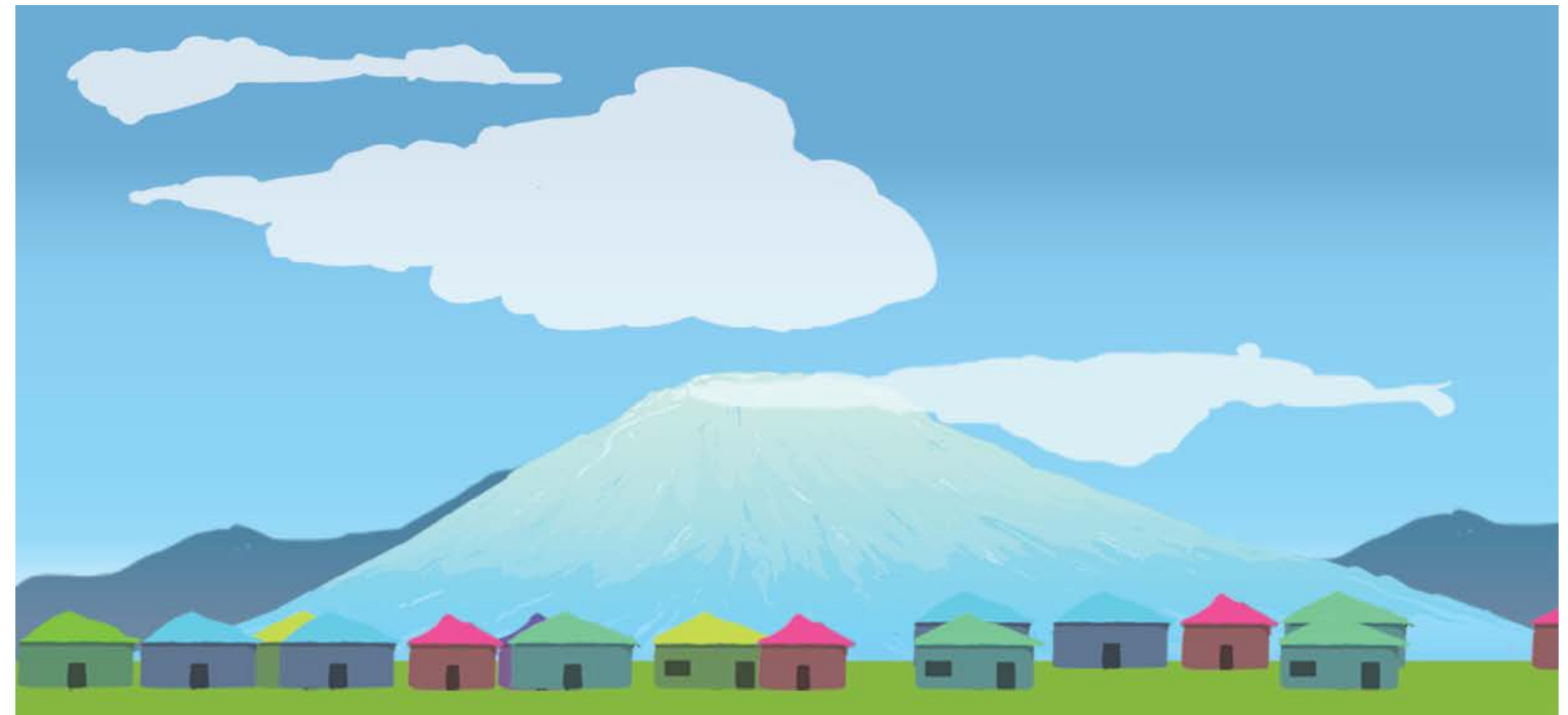

03

8

83

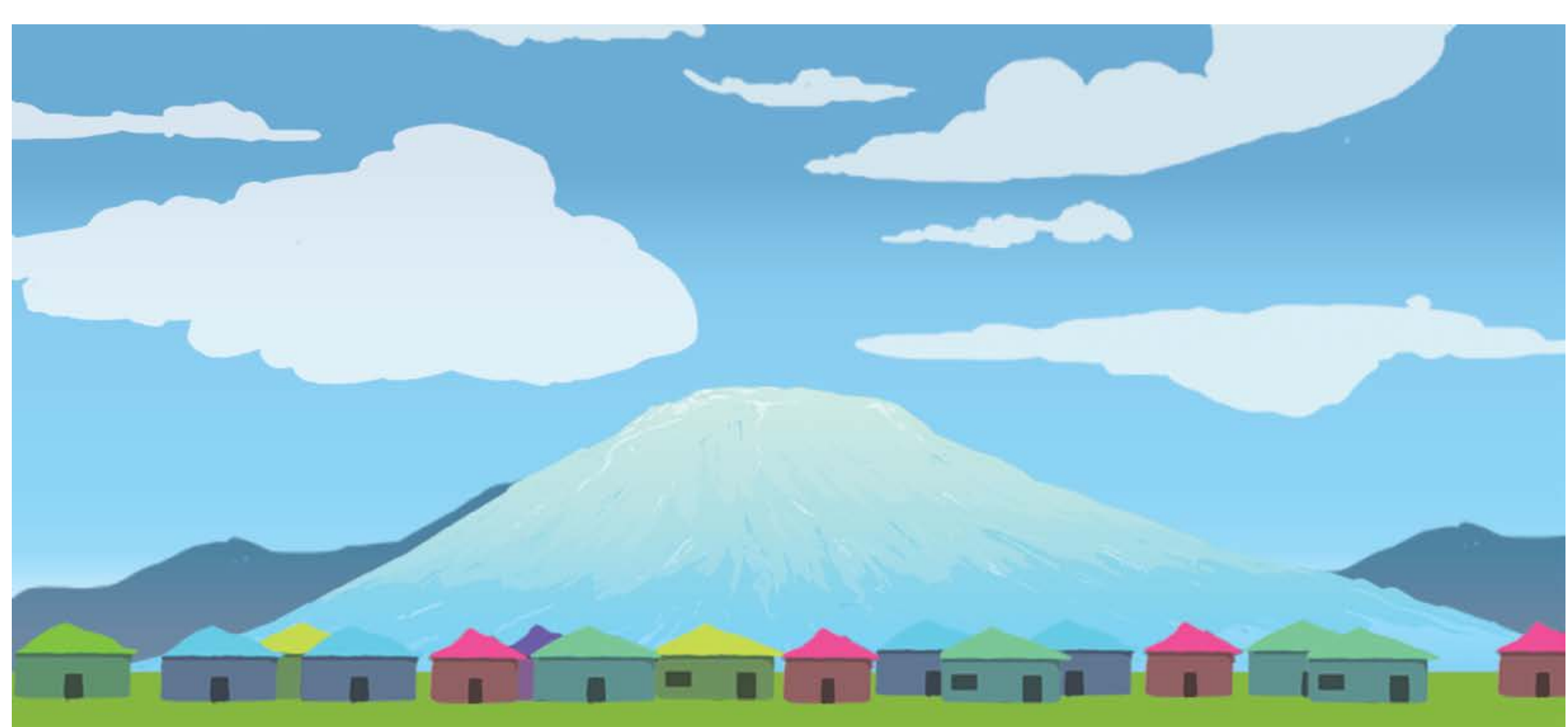

8

Fortalecimiento

de capacidades para

las adolescentes mujeres, orientado al desarrollo de Plan de Vida y Habilidades para la Vida.

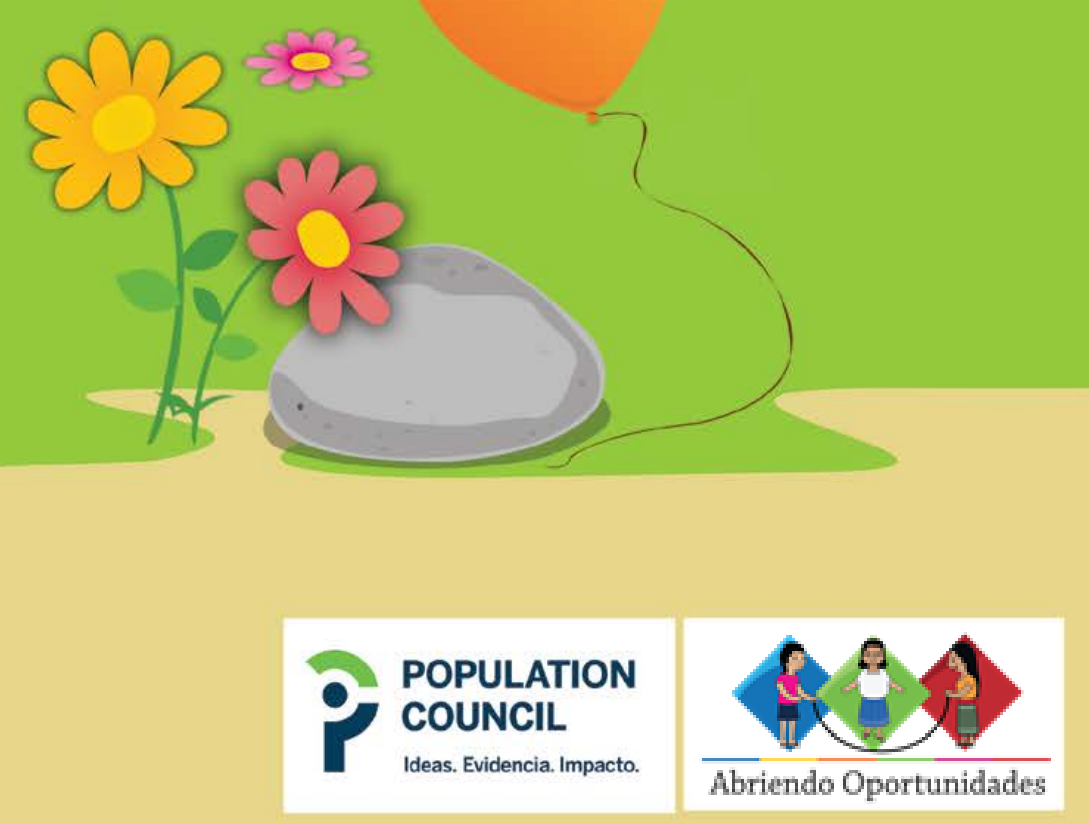

83

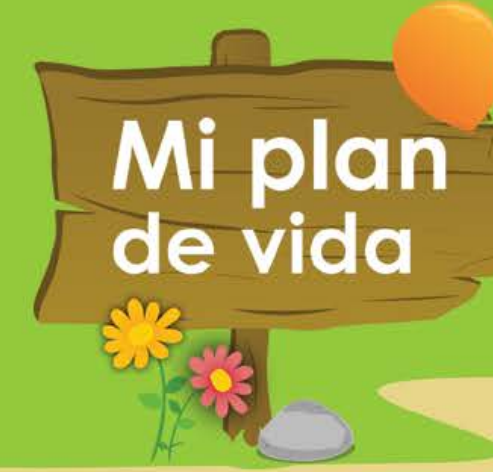


Population Council

Programa Abriendo Oportunidades $\AA$

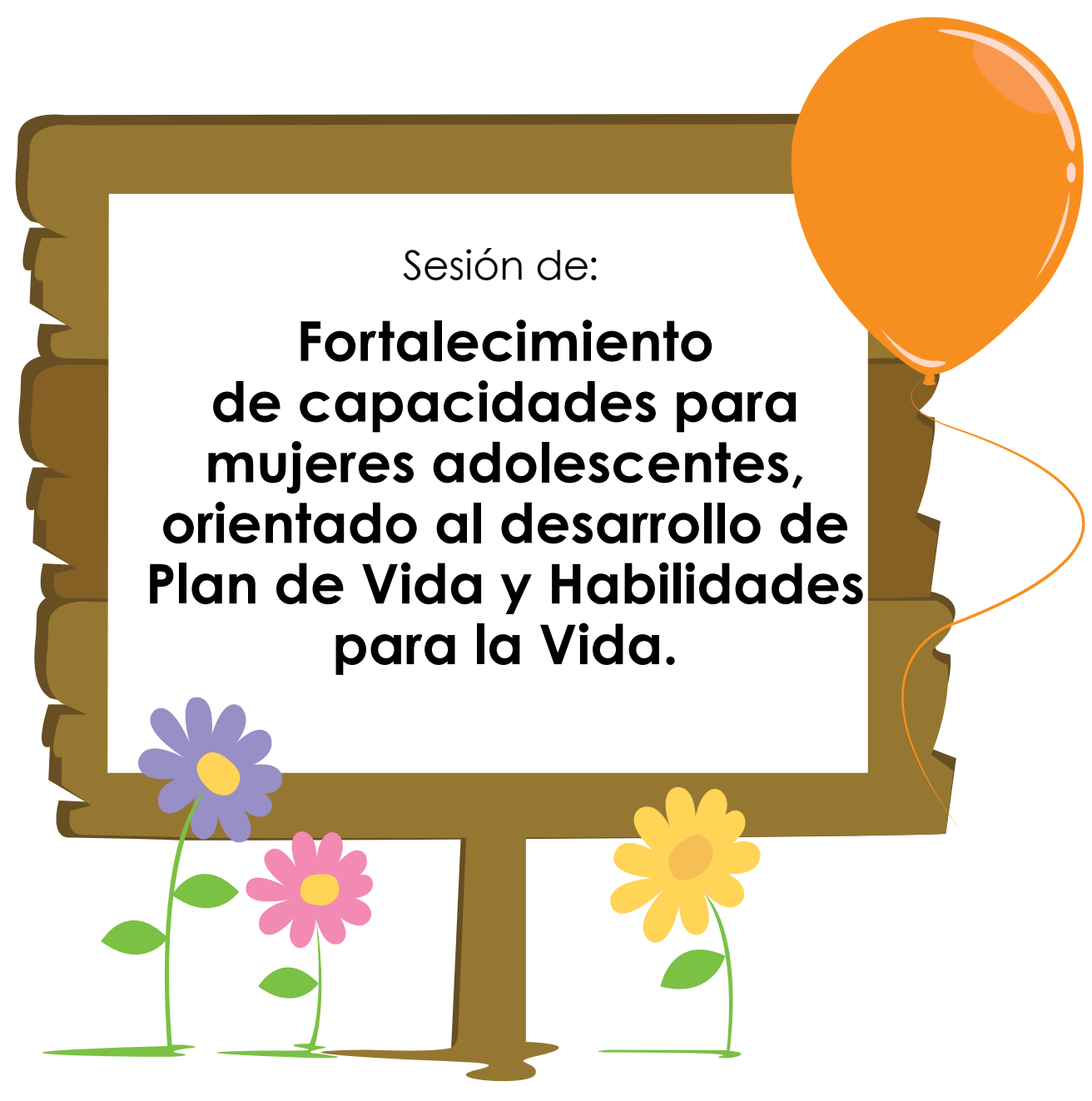

Aplicación a la vida:

Pida que cada una escriba una meta que tiene en su vida para cada uno de los siguientes aspectos: estudio, trabajo, familia ahorros y comunidad. Permita que compartan entre ellas.

a. Escribe tus datos en los espacios en el árbol

Escribe tus metas en cada una de las flechas

Escribe dentro de cada globo una acción para alcanzar tu c. meta.

Escribe dentro de cada piedra un reto que tendrás que enfrentar y superar para alcanzar cada meta

Escribe dentro de cada rótulo una fortaleza que te ayudará a alcanzar cada meta

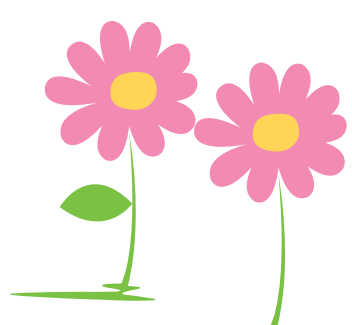

Puede pedir que en grupos pequeños o en plenaria compartan sus planes de vida. Si el grupo está de acuerdo es una oportunidad para presentarlos a la comunidad. Invite a las familias y autoridades locales a la próxima sesión para conocer los planes de vida de las niñas y que éstas les manifiesten lo que han aprendido asistiendo al grupo y lo que quieren lograr en sus vidas. 


- Relaciones sociales
- Familia
- Estudio
- Trabajo
- Ahorros
- Comunidad

Con esta oportunidad Flory ordenó sus ideas.

Sabía que quería, terminar el bachillerato y luego estudiar Psicología en la Universidad de San Carlos. No sabía cómo iba a lograrlo pero definitivamente no quería tener esposo ahora, más le gustaba pasar el tiempo con amigas y amigos. Mientras estudia en la Universidad podía trabajar para comprar libros, ayudar a su familia.

Por eso es necesario empezar a ahorrar ya.

Además de lo económico, estudir Psicología le permitiría ayudar a las mujeres de su comunidad cuando lo necesitaran.

La maestra les pidió que dibujaran su plan de vida como si fuera un camino, teniendo en cuenta:

- sus metas,

- los retos que enfrentarían

- sus fortalezas y

- las acciones a realizar

Fin.

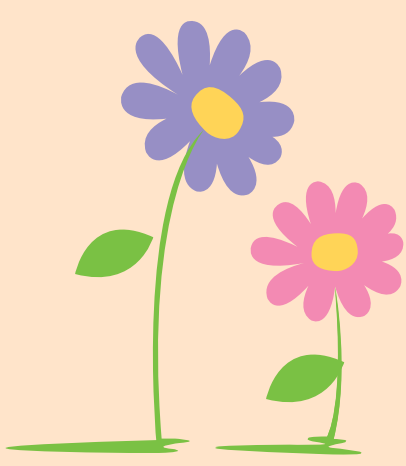

Fortalecimiento de capacidades para

las adolescentes mujeres,

orientado al desarrollo de Plan de Vida y

Habilidades para la Vida.

\section{Tabla para la facilitadora}

\begin{tabular}{|l|l|}
\hline $\begin{array}{l}\text { Al finalizar la } \\
\text { sesión las } \\
\text { participantes } \\
\text { habrán... }\end{array}$ & $\begin{array}{l}\text { Establecido } 5 \text { metas que tienen (estudio, trabajo, } \\
\text { familia, ahorros, comunidad). }\end{array}$ \\
$\begin{array}{l}\text { - Identificado personas que les apoyan. } \\
\text { - Identificado sus fortalezas para alcanzar sus metas. } \\
\text { - Identificado los retos que enfrentarán para alcanzar } \\
\text { sus metas. }\end{array}$ \\
\hline $\begin{array}{l}\text { Propuesto acciones a realizar para alcanzar sus metas } \\
\text { Conceptos }\end{array}$ & $\begin{array}{l}\text { Meta: algo que se quiere alcanzar en la vida. Puede ser } \\
\text { Clave }\end{array}$ \\
\hline
\end{tabular}

Clave

un objeto, una oportunidad, una acción.

Plan de vida: Los pasos para alcanzar las metas que se quieren alcanzar a corto, mediano y largo plazo. Considera las fortalezas, las oportunidades, las debilidades y los retos que se presentarán.

Fortaleza: cualidad que tiene una persona o un grupo que les hace destacar y ayuda en su desarrollo.

\begin{tabular}{l|l} 
Materiales & - Afiche del plan de vida \\
- Lapiz
\end{tabular}

Tiempo: 1.5 horas 


\section{Bienvenida:}

- Dé la bienvenida del día a las participantes, confirmando quiénes están presentes o ausentes. Cuénteles que usted tiene grandes planes para su vida y cuénteles uno. Dé oportunidad a que ellas también le cuenten un plan que tienen y uno que ya han cumplido.

\section{Introducción al tema:}

- Salgan al campo a jugar futbol, con una sola portería. Haga dos equipos entre las niñas y jueguen unos treinta minutos. Al terminar pregunte:

\section{¿Qué es lo que todas quieren hacer cuando juegan fútbol? Meter la pelota en la portería, en la meto.}

¿Qué esfuerzos hicieron para intentar llegar a la meta?

¿Qué debilidades les hizo más difícil alcanzar la meta?

¿Qué personas ayudaron a llegar a la meta?

¿Qué fortalezas tienen para llegar a la meta y jugar en equipo?

\section{Definir y teorizar:}

Cuénteles que en la vida también tenemos metas que queremos alcanzar y que al igual que en fútbol, hacemos muchos esfuerzos para alcanzarlas. Que en ese camino contamos con dos cosas para seguir la meta: las personas que hacen equipo y nos ayudan y las fortalezas personales que tenemos para alcanzar la meta.

Escriba la frase "Plan de Vida" y cuente qué es un plan de vidaPídales que en parejas comenten en qué se parece el plan de vida al juego de futbol.

Lleve el dibujo de una portería, un equipo, una jugadora con buena puntería y el equipo rival. Permita que ellas expliquen su comparación entre el plan de vida y el equipo de futbol.

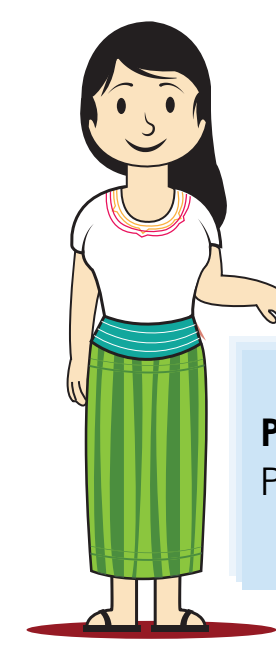

Plan de Vida

Plan del juego

Meta $=$

Portería

Personas que

apoyan =

Equipo de futbol

\section{Fortaleza}

Buena puntería

Reto $=$

Equipo rival

Cuente la historia de una joven que tenía deseos de hacer muchas cosas en su vida y que la van a ayudar a planificar cómo alcanzarlas. Entregue a cada una su afiche para que puedan leer juntas la historia.

\section{HISTORIA DE FLORY}

Desde que Flory nació, su mamá le cuenta sobre todas las cosas maravillosas que ella puede hacer en su vida gracias a sus fortalezas y el apoyo de su familia. Esta mañana en su calse, estaban hablando sobre tres compañeras que se fueron mojadas, estaban hablando sobre tres compañeras que se fueron mojadas,
fue muy tristes saber que sus amigas estaban en riesgo y en peligro. Flory no quería tener que irse a los Estados a trabajar pero conocía que muchas veces la pobreza les obliga. Su maestra conocía bien la pobreza en la que vivían sus alumnas y decidió dar tiempo para que cada alumna pudiera pensar en sus sueños y planes pensando en la estrategia que seguirían para alcanzarlos.

El primer paso, es identificar sus metas que tenía. Para Flory fue muy fácil, tenía tantas que ocuparía toda una página. La maestra vio que muchas como ella escribían y escribían entonces les pidió poner una meta por algunos aspectos: 